\title{
Three-Input Molecular Keypad Lock Based on a Norbornadiene- Quadricyclane Photoswitch
}

\author{
Ambra Dreos, Zhihang Wang, Behabitu E. Tebikachew, Kasper Moth-Poulsen,*(i) \\ and Joakim Andréasson*(1)
}

Department of Chemistry and Chemical Engineering, Chalmers University of Technology, 41296 Gothenburg, Sweden

\section{Supporting Information}

ABSTRACT: The photo- and acidochromic properties of a new generation norbornadiene derivative have been harnessed for the realization of a three-input keypad lock, where a specific sequence of inputs induces a unique output. Reversible quadricyclane/norbornadiene photoisomerization is reported, and this rare feature allows the first example of a norbornadiene-based molecular logic system. The function of this system is clearly rationalized in terms of the interconversion scheme and the absorption spectra of the involved species.

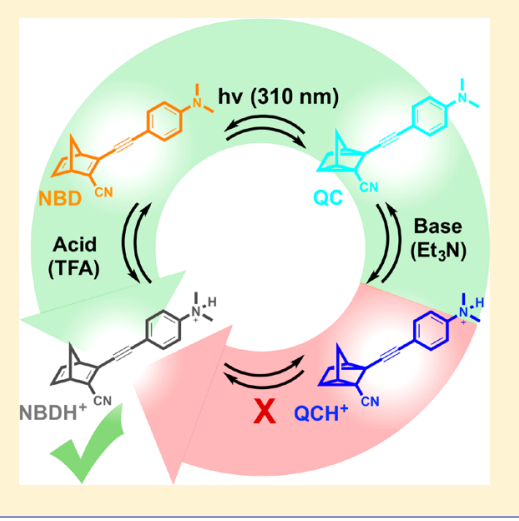

$\mathrm{M}$ olecular logic and information processing is a research field in which molecules are designed to emulate functions conventionally performed by electronic hardware. ${ }^{1-3}$ While the latter is voltage controlled, the molecular versions are not being restricted by this boundary condition. Instead, the researcher can choose the inputs and the outputs freely, to fit the intended use. ${ }^{4,5}$ Photonic and chemical (metal cations, DNA oligomers, acid/base, etc.) inputs enjoy preference, whereas the outputs are typically read by spectroscopic means (e.g., UV-vis absorption or fluorescence emission). The pioneering example was reported by de Silva in $1993 .{ }^{6}$ An anthracene fluorophore equipped with two receptors was designed to display strong emission (output) only upon the presence of both $\mathrm{Na}^{+}$ions and protons (inputs), implying the realization of the first molecular logic AND gate. Since then, more and more complex functions have been mimicked by molecule-based systems, often requiring a multitude of inputs and outputs. ${ }^{7,8}$ Special attention has been given to the keypad lock, a device where not only the correct input combination matters but also the sequence in which the inputs are applied. ${ }^{9,10}$ Hence, the keypad locks are so-called sequential logic devices. The first example of a molecular version was realized by Shanzer and Margulies in 2007. ${ }^{11}$ Several alternative approaches have been presented, including systems with input combinations of all-photonic, all-chemical, or mixed character. ${ }^{12-18}$ In the all-photonic case, photochromic systems are par excellence candidates as the spectral changes required to perform the desired logic function are conveniently triggered by photonic stimuli. Photoswitches from several different photochromic families have been used including spiropyrans, diarylethenes, and fulgimides. ${ }^{19}$ Here, we present the first example of a molecular logic device based on a norborna- diene-quadricyclane photoswitch. Using photons and acid/ base chemistry, it performs the function of a three-input keypad lock.

Norbornadiene is a bicyclic organic compound which upon irradiation with UV light is converted to the highly strained and saturated quadricyclane isomer. ${ }^{20-22}$ Back-isomerization to the norbornadiene form has been observed thermally, electrochemically, catalytically, and photonically. ${ }^{21,23-26}$ Norbornadienes have been widely studied over the years for solar thermal energy storage applications. ${ }^{27-30}$ They have also been studied as molecular electronic components. ${ }^{31}$ Synthesis of norbornadiene derivatives has been explored in the past for their importance as synthetic intermediates, and for other applications. $^{32}$ Recently, the research area has seen a resurgence, thanks to the contributions of modern coupling reactions and advanced computational tools. ${ }^{33-39}$ Most often, the unsubstituted norbornadiene is not ideal for these purposes. Thus, different molecular modifications have been explored to optimize the performance, including the introduction of electron donor and acceptor groups on one or both double bonds. ${ }^{27,29,30,33,34}$

Norbornadiene derivative NBD in Figure 1 has been previously synthesized. ${ }^{34}$ The synthesis starts from the commercially available norbornadiene, and it is straightforward and efficient over only three steps. It is a versatile derivative with interesting properties that have been already partially characterized and exploited in previous works but will be here discussed in depth and further expanded, with the introduction

Received: August 20, 2018

Accepted: October 8, 2018

Published: October 8, 2018 

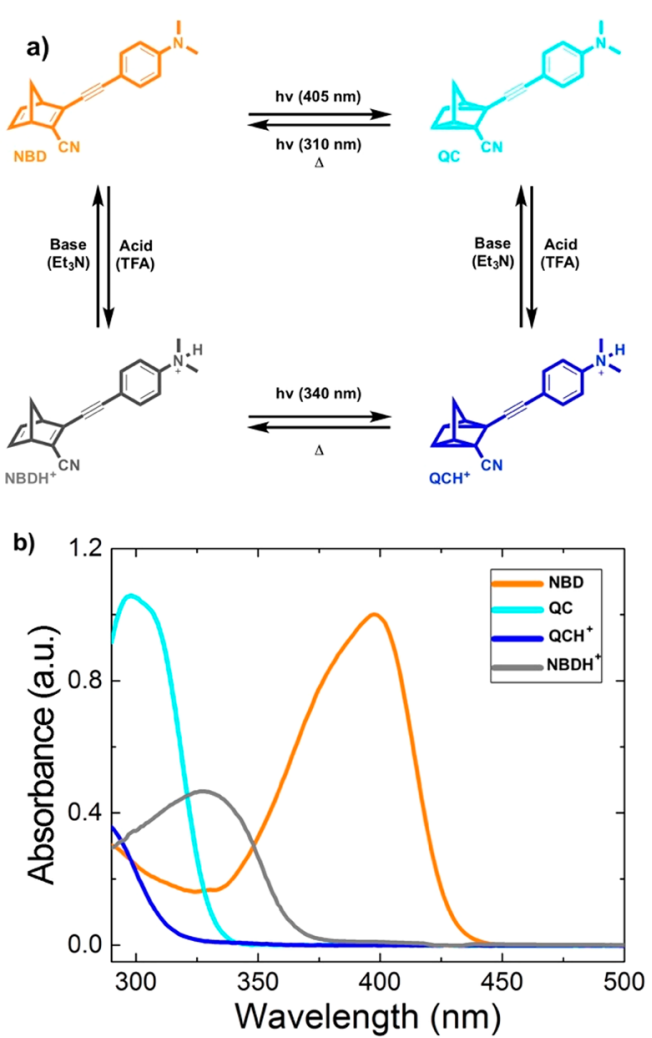

Figure 1. (a) Interconversion scheme for the four molecular species $\mathrm{NBD}, \mathrm{QC}, \mathrm{NBDH}^{+}$, and $\mathrm{QCH}^{+}$. Irradiation at $405 \mathrm{~nm}$ photoconverts NBD to QC, while QC can isomerize back to NBD photochemically $(310 \mathrm{~nm})$ or thermally (over hours at room temperature). Both NBD and $\mathrm{QC}$ can be protonated using for example trifluoroacetic acid (TFA), and $\mathrm{NBDH}^{+}$and $\mathrm{QCH}^{+}$can be deprotonated using a base as triethylamine $\left(\mathrm{Et}_{3} \mathrm{~N}\right)$. Photoisomerization of $\mathrm{NBDH}^{+}$to $\mathrm{QCH}^{+}$is observed upon irradiation at $340 \mathrm{~nm}$, whereas $\mathrm{QCH}^{+}$is thermally isomerized to $\mathrm{NBDH}^{+}$(over hours at room temperature). (b) UV-vis absorption spectra of the four implicated species NBD, $\mathrm{QC}, \mathrm{NBDH}^{+}$, and $\mathrm{QCH}^{+}$. All samples prepared as toluene solutions.

of novel features and applications. The structural rearrangement responsible for the isomerization is the breaking of the double bonds to form the corresponding single bonds (Figure 1a), which interrupts the conjugation in the donor-acceptor system and therefore significantly blue-shifts the absorption of the QC isomer (Figure 1b). While unsubstituted norbornadiene does not absorb above $300 \mathrm{~nm}$, NBD, thanks to the donor-acceptor groups introduced on the double bond, absorbs up to around $460 \mathrm{~nm}$ with absorption maximum at $398 \mathrm{~nm} .{ }^{34}$ Upon light exposure at wavelengths between ca. 350 and $425 \mathrm{~nm}$ it photoisomerizes to the corresponding quadricyclane (QC) form with a measured quantum yield of $28 \%{ }^{34}$ As indicated above, breaking the conjugation of the push-pull system blue-shifts the absorption substantially. The QC absorption has an onset at $344 \mathrm{~nm}$ and an absorption maximum at $298 \mathrm{~nm}$. The back-isomerization from QC to NBD has been observed to be induced thermally (time constant of $7.3 \mathrm{~h}$ at $25^{\circ} \mathrm{C}$ in toluene). Quadricyclane units incorporated into polymers have been reported to undergo photoinduced conversion to the norbornadiene isomer, which was achieved by irradiation with short-wave UV light. ${ }^{23,24}$ Quadricyclane to norbornadiene photoisomerization in the newer generations of derivatives with red-shifted absorption is, however, extremely rare. ${ }^{40}$ Despite this fact, we decided to irradiate QC with light at $310 \mathrm{~nm}$, and to our surprise it readily and almost completely converted to NBD. This feature is of great interest, since it allows conversion of QC to NBD on a much shorter time scale than the thermal process. Being able to convert readily and on demand between QC and NBD opens the utilization of this system to new applications where a fast and clean conversion between the species is needed. The quantum yield of the $\mathrm{QC} \rightarrow \mathrm{NBD}$ photoisomerization was measured as $53 \%$ at $300 \mathrm{~nm}$ (see Supporting Information page 9 for more information). The fatigue resistance of NBD-QC isomers during photothermal and all-photonic processes was tested, showing excellent robustness with no or small degradation over about 100 cycles (see our previous work ${ }^{37}$ or Supporting Information page 10 for more information).

This system has more interesting features; protonation of the methylamino group allows for the formation of two additional species: $\mathrm{NBDH}^{+}$and $\mathrm{QCH}^{+}$. Since the protonation of the amino group reduces the electron-donating character, $\mathrm{NBDH}^{+}$and $\mathrm{QCH}^{+}$are expected to have a more blue-shifted absorption compared to that of the nonprotonated species. This is indeed also observed experimentally (Figure 1b). The structures and the interconversion scheme of all four implicated forms are shown in Figure 1a. Here, irradiation of NBD at $405 \mathrm{~nm}$ was used to induce full photoisomerization to QC, while irradiation at $310 \mathrm{~nm}$ allowed for almost complete back-photoisomerization. Partial isomerization of $\mathrm{NBDH}^{+}$to $\mathrm{QCH}^{+}$was also observed when the sample was irradiated at $340 \mathrm{~nm}$. All photoinduced processes proceed readily at modest light intensities (25-870 $\mathrm{mW}$, less than a minute irradiation). This should be contrasted with the thermal isomerizations occurring on the time scale of several hours (time constants of 7.3 and $2.5 \mathrm{~h}$ at room temperature for $\mathrm{QC} \rightarrow \mathrm{NBD}$ and $\mathrm{QCH}^{+}$ $\rightarrow \mathrm{NBDH}^{+}$, respectively; see also Supporting Information page 5).

After identifying all the states and interconversions in the system, the function of NBD as a three-input keypad lock is easily rationalized. We define the three inputs as acid (a), base (b), and light at $310 \mathrm{~nm}$ (UV). From Figure 1a, it is clear that that there is only one input sequence that interconverts $\mathrm{QCH}^{+}$ to $\mathrm{NBDH}^{+}$, and therefore, a unique output is obtained only by using a unique input sequence, so that the molecular system can serve as a keypad lock. Thus, we define $\mathrm{QCH}^{+}$as the initial state. $\mathrm{NBDH}^{+}$has a characteristic absorption band centered at around $330 \mathrm{~nm}$, a wavelength where all other forms display no or low absorption only. The absorbance at $330 \mathrm{~nm}$ is therefore chosen as the output.

Exposing $\mathrm{QCH}^{+}$to $310 \mathrm{~nm}$ UV light does not significantly affect the isomeric distribution between $\mathrm{QCH}^{+}$and $\mathrm{NBDH}^{+}$.

Table 1. All Possible Sequential Combinations of the Inputs, $a=$ Acid, $b=$ Base, UV = Irradiation at $310 \mathrm{~nm}$

\begin{tabular}{|c|c|c|c|c|c|c|c|c|c|c|c|c|c|c|}
\hline 1 & 2 & 3 & 4 & 5 & 6 & 7 & 8 & 9 & 10 & 11 & 12 & 13 & 14 & 15 \\
\hline a & $a$ & $\mathrm{~b}$ & $\mathrm{~b}$ & UV & UV & $\mathrm{a}$ & $\mathrm{b}$ & a & UV & $\mathrm{b}$ & UV & $\mathrm{a}$ & $\mathrm{b}$ & UV \\
\hline $\mathrm{b}$ & UV & a & UV & a & $\mathrm{b}$ & $\mathrm{b}$ & a & UV & a & UV & $\mathrm{b}$ & & & \\
\hline
\end{tabular}


Table 2. Predicted Resulting Species after Applying the Input Sequences to the Initial State $\mathrm{QCH}^{+a}$

\begin{tabular}{|c|c|c|c|c|c|c|c|c|c|c|c|c|c|c|}
\hline 1 & 2 & 3 & 4 & 5 & 6 & 7 & 8 & 9 & 10 & 11 & 12 & 13 & 14 & 15 \\
\hline $\mathbf{Q C H}^{+}$ & $\mathrm{QCH}^{+}$ & $\mathrm{QCH}^{+}$ & $\mathbf{Q C H}^{+}$ & $\mathrm{QCH}^{+}$ & $\mathrm{QCH}^{+}$ & $\mathrm{QCH}^{+}$ & $\mathbf{Q C H}^{+}$ & $\mathrm{QCH}^{+}$ & $\mathbf{Q C H}^{+}$ & $\mathrm{QCH}^{+}$ & $\mathrm{QCH}^{+}$ & $\mathrm{QCH}^{+}$ & $\mathbf{Q C H}^{+}$ & $\mathbf{Q C H}^{+}$ \\
\hline $\mathrm{QCH}^{+}$ & $\mathbf{Q C H}^{+}$ & QC & QC & $\mathbf{Q C H}^{+}$ & $\mathbf{Q C H}^{+}$ & $\mathbf{Q C H}^{+}$ & QC & $\mathbf{Q C H}^{+}$ & $\mathbf{Q C H}^{+}$ & QC & $\mathbf{Q C H}^{+}$ & $\mathbf{Q C H}^{+}$ & QC & $\mathbf{Q C H}^{+}$ \\
\hline QC & $\mathbf{Q C H}^{+}$ & $\mathbf{Q C H}^{+}$ & NBD & $\mathbf{Q C H}^{+}$ & QC & QC & $\mathbf{Q C H}^{+}$ & $\mathbf{Q C H}^{+}$ & $\mathbf{Q C H}^{+}$ & NBD & QC & & & \\
\hline
\end{tabular}

${ }^{a}$ Number 4 is the only expected sequence to produce $\mathrm{NBDH}^{+}$as the main final species indicated at the bottom line (color coded).

Instead, $\mathrm{QCH}^{+}$must first be deprotonated by the addition of base (triethylamine, $\mathrm{Et}_{3} \mathrm{~N}$, added in excess) to yield QC. QC is isomerized to NBD by $310 \mathrm{~nm}$ UV for about $60 \mathrm{~s}$. Subsequent addition of acid (trifluoroacetic acid, TFA, added in excess) results in the formation of $\mathrm{NBDH}^{+}$displaying strong absorption at $330 \mathrm{~nm}$, switching the output to the on-state, equivalent to opening the lock. Thus, due to the intrinsically much slower thermal isomerization processes, there is no other way to produce $\mathrm{NBDH}^{+}$on this time scale than the input order b, UV, a (addition of $\mathrm{Et}_{3} \mathrm{~N}$, irradiation at $310 \mathrm{~nm}$, and addition of TFA).

In order to experimentally verify the above-mentioned performance, all the permutations generated by combinations of one, two, or all three inputs (Table 1) were subjected to the system in toluene solution. Before each of the input sequences was applied, the initial state $\mathrm{QCH}^{+}$was prepared from the assynthesized NBD form by photoisomerization at $405 \mathrm{~nm}$ followed by addition of TFA. The predicted resulting species after applying the 15 input sequences are shown in Table 2. Indeed, this is also experimentally verified from the corresponding absorption spectra (Supporting Information pages $6-7$ ).

Extracting the absorbance values at the output wavelength $330 \mathrm{~nm}$ yields the graph shown in Figure 2. Only one out of

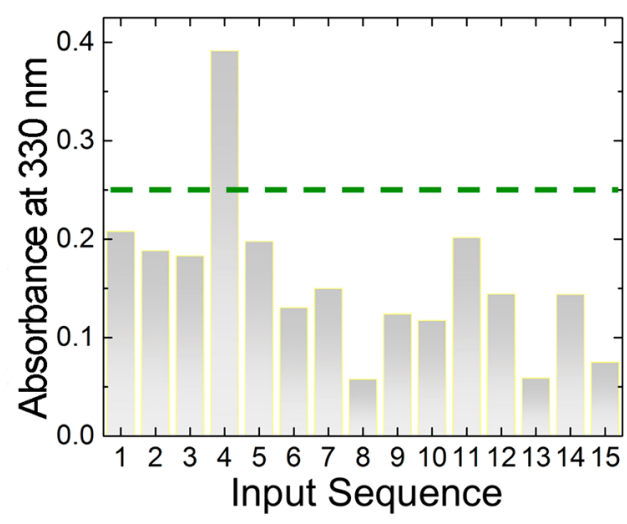

Figure 2. Output readings of the absorbance at $330 \mathrm{~nm}$ after applying sequences $1-15$. Number 4 is the only sequence that yields an absorbance above the threshold level, arbitrarily set at $A=0.25$.

the 15 possible input combinations, namely, the anticipated bUV-a (input sequence 4), yields an absorbance above the threshold level set to 0.25 . These proof of principle experiments show that it is possible to use the described system as a three-input molecular keypad lock, where the output signal for the correct input sequence is at least 1.6 times higher (equivalent to the dynamic range) than for all others.
The presented NBD derivative has already many advantages compared to those of previously reported systems, such as the ease of synthesis ${ }^{34}$ and the demonstrated robustness over many cycles. ${ }^{37}$ Moreover, NBDs synthesis and physical and chemical behaviors and properties are widely studied and quite well understood, ${ }^{29,32,35,36,38}$ implying the facilitation of carefully designing molecular systems with the desired properties and predictable physicochemical behavior. Some limitations and challenges can be identified. For example, the described molecular system is based on metastable states, displaying thermal isomerizations on the time scale of 3-7 h. In the operation of NBD as a keypad lock, however, the time required to apply the photonic and chemical inputs is below $1 \mathrm{~min}$, and in this time the thermal isomerization of the metastable species is minimal $(<4 \%)$. A universal reset operation can be suggested, where the sample is basified to induce deprotonation, and heat is applied to induce back-conversion of any QC to the stable form NBD. If multiple cycles are considered, however, the use of chemical inputs results in chemical byproducts and eventual dilution, interfering with the intended function.

To conclude, we have demonstrated the first example of a norbornadiene-quadricyclane photoswitch in the context of molecular logic. Using photons and acid/base as inputs, it performs as a three-input keypad lock. The function is easily rationalized from the underlying isomerization and protonation/deprotonation scheme. The advantage of using NBD derivatives is that NBD chemical modifications have been abundantly explored in the past and more recently, 29,30,32,34,39 which allows us to access a wide library of compounds and synthetic methods. For these reasons there are certainly great opportunities for improved design aiming at more streamlined keypad locks, or complex multiswitch systems allowing for even more sophisticated logic functions to be performed.

\section{ASSOCIATED CONTENT}

\section{S Supporting Information}

The Supporting Information is available free of charge on the ACS Publications website at DOI: 10.1021/acs.jpclett.8b02567.

General methods, NMR spectra, kinetics of thermal back-isomerization of $\mathrm{QCH}^{+}$to $\mathrm{NBDH}^{+}, \mathrm{UV}-$ vis spectra during input sequences $1-15$, output readings, quantum yield of photoisomerization, and fatigue test of NBD-QC photoswitching (PDF)

\section{AUTHOR INFORMATION}

\section{Corresponding Authors}

*K. Moth-Poulsen. E-mail: kasper.moth-poulsen@chalmers.se. 
*J. Andréasson. E-mail: a-son@chalmers.se.

\section{ORCID}

Kasper Moth-Poulsen: 0000-0003-4018-4927

Joakim Andréasson: 0000-0003-4695-7943

\section{Notes}

The authors declare no competing financial interest.

\section{ACKNOWLEDGMENTS}

The Swedish Strategic Research Foundation SSF, the Swedish Research Council VR, the European Research Council ERC, and the Knut and Alice Wallenberg Foundation are acknowledged for financial support. We acknowledge M. Quant for providing the norbornadiene used in this work and $\mathrm{K}$. Börjesson for valuable discussion. There are no conflicts to declare.

\section{REFERENCES}

(1) Pilarczyk, K.; Wlaźlak, E.; Przyczyna, D.; Blachecki, A.; Podborska, A.; Anathasiou, V.; Konkoli, Z.; Szaciłowski, K. Molecules, Semiconductors, Light and Information: Towards Future Sensing and Computing Paradigms. Coord. Chem. Rev. 2018, 365, 23-40.

(2) Andréasson, J.; Pischel, U. Molecules with a Sense of Logic: A Progress Report. Chem. Soc. Rev. 2015, 44, 1053-1069.

(3) Erbas-Cakmak, S.; Kolemen, S.; Sedgwick, A. C.; Gunnlaugsson, T.; James, T. D.; Yoon, J.; Akkaya, E. U. Molecular Logic Gates: The Past, Present and Future. Chem. Soc. Rev. 2018, 47, 2228-2248.

(4) Balzani, V.; Credi, A.; Venturi, M. Processing Energy and Signals by Molecular and Supramolecular Systems. Chem. - Eur. J. 2008, 14, 26-39.

(5) Magri, D. C.; Camilleri Fava, M.; Mallia, C. J. A Sodium-Enabled 'Pourbaix Sensor': A Three-Input AND Logic Gate as a 'Lab-on-aMolecule' for Monitoring $\mathrm{Na}^{+}, \mathrm{pH}$ and $\mathrm{pE}$. Chem. Commun. 2014, 50, 1009-1011.

(6) De Silva, P. A.; Gunaratne, N. H. Q.; McCoy, C. P. A Molecular Photoionic and Gate Based on Fluorescent Signalling. Nature 1993, 364, 42-44.

(7) Andréasson, J.; Pischel, U.; Straight, S. D.; Moore, T. A.; Moore, A. L.; Gust, D. All-Photonic Multifunctional Molecular Logic Device. J. Am. Chem. Soc. 2011, 133, 11641-11648.

(8) Chen, S.; Yang, Y.; Wu, Y.; Tian, H.; Zhu, W. Multi-Addressable Photochromic Terarylene Containing Benzo[b]Thiophene-1,1-Dioxide Unit as Ethene Bridge: Multifunctional Molecular Logic Gates on Unimolecular Platform. J. Mater. Chem. 2012, 22, 5486-5494.

(9) Andréasson, J.; Pischel, U. Molecules for Security Measures: From Keypad Locks to Advanced Communication Protocols. Chem. Soc. Rev. 2018, 47, 2266-2279.

(10) Lustgarten, O.; Motiei, L.; Margulies, D. User Authorization at the Molecular Scale. ChemPhysChem 2017, 18, 1678-1687.

(11) Margulies, D.; Felder, C. E.; Melman, G.; Shanzer, A. A Molecular Keypad Lock: A Photochemical Device Capable of Authorizing Password Entries. J. Am. Chem. Soc. 2007, 129, 347-354.

(12) Kumar, S.; Luxami, V.; Saini, R.; Kaur, D. Superimposed Molecular Keypad Lock and Half-Subtractor Implications in a Single Fluorophore. Chem. Commun. 2009, 0, 3044-3046.

(13) Sun, W.; Zhou, C.; Xu, C. H.; Fang, C. J.; Zhang, C.; Li, Z. X.; Yan, C. H. A Fluorescent-Switch-Based Computing Platform in Defending Information Risk. Chem. - Eur. J. 2008, 14, 6342-6351.

(14) Andréasson, J.; Straight, S. D.; Moore, T. A.; Moore, A. L.; Gust, D. An All-Photonic Molecular Keypad Lock. Chem. - Eur. J. 2009, 15, 3936-3939.

(15) Kink, F.; Collado, M. P.; Wiedbrauk, S.; Mayer, P.; Dube, H. Bistable Photoswitching of Hemithioindigo with Green and Red Light: Entry Point to Advanced Molecular Digital Information Processing. Chem. - Eur. J. 2017, 23, 6237-6243.

(16) Strack, G.; Ornatska, M.; Pita, M.; Katz, E. Biocomputing Security System: Concatenated Enzyme-Based Logic Gates Operating as a Biomolecular Keypad Lock. J. Am. Chem. Soc. 2008, 130, 42344235.

(17) Zhu, J.; Yang, X.; Zhang, L.; Zhang, L.; Lou, B.; Dong, S.; Wang, E. A Visible Multi-Digit DNA Keypad Lock Based on Split GQuadruplex DNAzyme and Silver Microspheres. Chem. Commun. 2013, 49, 5459-5461.

(18) Jiang, X. J.; Ng, D. K. P. Sequential Logic Operations with a Molecular Keypad Lock with Four Inputs and Dual Fluorescence Outputs. Angew. Chem., Int. Ed. 2014, 53, 10481-10484.

(19) Irie, M. Diarylethenes for Memories and Switches. Chem. Rev. 2000, 100, 1685-1716.

(20) Cristol, S. J.; Snell, R. L. Bridged Polycyclic Compounds. VI. The Photoisomerization of Bicyclo [2,2,1] Hepta-2,5-Diene-2,3Dicarboxylic Acid to Quadricyclo [2,2,1,0,0] Heptane-2,3-Dicarboxylic Acid. J. Am. Chem. Soc. 1958, 80, 1950-1952.

(21) Hammond, G. S.; Turro, N.; Fischer, A. Photosensitized Cycloaddition Reactions. J. Am. Chem. Soc. 1961, 83, 4674-4675.

(22) Hammond, G. S.; Wyatt, P.; DeBoer, D. C.; Turro, N. J. Photosensitized Isomerization Involving Saturated Centers. J. Am. Chem. Soc. 1964, 86, 2532-2533.

(23) Brummel, O.; Waidhas, F.; Bauer, U.; Wu, Y.; Bochmann, S.; Steinrück, H. P.; Papp, C.; Bachmann, J.; Libuda, J. Photochemical Energy Storage and Electrochemically Triggered Energy Release in the Norbornadiene-Quadricyclane System: UV Photochemistry and IR Spectroelectrochemistry in a Combined Experiment. J. Phys. Chem. Lett. 2017, 8, 2819-2825.

(24) Miki, S.; Maruyama, T.; Ohno, T.; Tohma, T.; Toyama, S.; Yoshida, Z. Alumina-Anchored Cobalt(II) Schiff Base Catalyst for the Isomerization of Trimethyldicyanoquadricyclane to the Norbornadiene. Chem. Lett. 1988, 17, 861-864.

(25) Kamogawa, H.; Yamada, M. Photoresponsive Vinyl Polymer Bearing Norbornadiene as a Pendant Group. Macromolecules 1988, $21,918-923$.

(26) Nishikubo, T.; Kameyama, A.; Kishi, K.; Kawashima, T.; Fujiwara, T.; Hijikata, C. Synthesis of New Photoresponsive Polymers Bearing Norbornadiene Moieties by Selective Cationic Polymerization of 2-[[(3-Phenyl-2,5-Norbornadienyl)-2-Carbonyl]Oxy]Ethyl Vinyl Ether and Photochemical Reaction of the Resulting Polymers. Macromolecules 1992, 25, 4469-4475.

(27) Yoshida, Z. New Molecular Energy Storage Systems. J. Photochem. 1985, 29, 27-40.

(28) Bren, V. A.; Dubonosov, A. D.; Minkin, V. I.; Chernoivanov, V. A. Norbornadiene-Quadricyclane - an Effective Molecular System for the Storage of Solar Energy. Russ. Chem. Rev. 1991, 60, 451.

(29) Dubonosov, A. D.; Bren, V. A.; Chernoivanov, V. A. Norbornadiene-Quadricyclane as an Abiotic System for the Storage of Solar Energy. Russ. Chem. Rev. 2002, 71, 917-927.

(30) Lennartson, A.; Roffey, A.; Moth-Poulsen, K. Designing Photoswitches for Molecular Solar Thermal Energy Storage. Tetrahedron Lett. 2015, 56, 1457-1465.

(31) Tebikachew, B. E.; Li, H. B.; Pirrotta, A.; Börjesson, K.; Solomon, G. C.; Hihath, J.; Moth-Poulsen, K. Effect of Ring Strain on the Charge Transport of a Robust Norbornadiene-QuadricyclaneBased Molecular Photoswitch. J. Phys. Chem. C 2017, 121, 70947100.

(32) Jack, K.; Machin, B.; Tigchelaar, A.; Tam, W. Synthesis of Substituted Norbornadienes. Curr. Org. Synth. 2013, 10, 584-630.

(33) Gray, V.; Lennartson, A.; Ratanalert, P.; Börjesson, K.; MothPoulsen, K. Diaryl-Substituted Norbornadienes with Red-Shifted Absorption for Molecular Solar Thermal Energy Storage. Chem. Commun. 2014, 50, 5330-5332.

(34) Quant, M.; Lennartson, A.; Dreos, A.; Kuisma, M.; Erhart, P.; Börjesson, K.; Moth-Poulsen, K. Low Molecular Weight Norbornadiene Derivatives for Molecular Solar-Thermal Energy Storage. Chem. - Eur. J. 2016, 22, 13265-13274.

(35) Kuisma, M. J.; Lundin, A. M.; Moth-Poulsen, K.; Hyldgaard, P.; Erhart, P. Comparative Ab-Initio Study of Substituted Norbornadiene-Quadricyclane Compounds for Solar Thermal Storage. J. Phys. Chem. C 2016, 120, 3635-3645. 
(36) Kuisma, M.; Lundin, A.; Moth-Poulsen, K.; Hyldgaard, P.; Erhart, P. Optimization of Norbornadiene Compounds for Solar Thermal Storage by First-Principles Calculations. ChemSusChem 2016, 9, 1786-1794.

(37) Dreos, A.; Börjesson, K.; Wang, Z.; Roffey, A.; Norwood, Z.; Kushnir, D.; Moth-Poulsen, K. Exploring the Potential of a Hybrid Device Combining Solar Water Heating and Molecular Solar Thermal Energy Storage. Energy Environ. Sci. 2017, 10, 728-734.

(38) Jorner, K.; Dreos, A.; Emanuelsson, R.; Ouissam, E. B.; Fdez Galvan, I.; Börjesson, K.; Feixas, F.; Lindh, R.; Burkhard, Z.; MothPoulsen, K.; et al. Unraveling Factors Leading to Efficient Norbornadiene-Quadricyclane Molecular Solar- Thermal Energy Storage Systems. J. Mater. Chem. A 2017, 5, 12369-12378.

(39) Mansø, M.; Petersen, A. U.; Wang, Z.; Erhart, P.; Nielsen, M. B.; Moth-Poulsen, K. Molecular Solar Thermal Energy Storage in Photoswitch Oligomers Increases Energy Densities and Storage Times. Nat. Commun. 2018, 9, 1945.

(40) Tebikachew, B. E.; Edhborg, F.; Kann, N.; Albinsson, B.; MothPoulsen, K. Turn-off Mode Fluorescent Norbornadiene-Based Photoswitches. Phys. Chem. Chem. Phys. 2018, 20, 23195-23201. 